Research Article

\title{
Application of a New Generalized Fractional Derivative and Rank of Control Measures on Cholera Transmission Dynamics
}

\author{
Kumama Regassa Cheneke $\mathbb{D}$, Koya Purnachandra Rao $i$, \\ and Geremew Kenassa Edessa \\ Department of Mathematics, Wollega University, Nekemte, Ethiopia \\ Correspondence should be addressed to Kumama Regassa Cheneke; kumamaregassa@gmail.com
}

Received 11 August 2021; Revised 15 October 2021; Accepted 16 October 2021; Published 2 November 2021

Academic Editor: Vladimir Mityushev

Copyright (C) 2021 Kumama Regassa Cheneke et al. This is an open access article distributed under the Creative Commons Attribution License, which permits unrestricted use, distribution, and reproduction in any medium, provided the original work is properly cited.

\begin{abstract}
In this study, the mathematical model of the cholera epidemic is formulated and analyzed to show the impact of Vibrio cholerae in reserved freshwater. Moreover, the results obtained from applying the new fractional derivative method show that, as the order of the fractional derivative increases, cholera-preventing behaviors also increase. Also, the finding of our study shows that the dynamics of Vibrio cholerae can be controlled if continuous treatment is applied in reserved freshwater used for drinking purposes so that the intrinsic growth rate of Vibrio cholerae in water is less than the natural death of Vibrio cholerae. We have applied the stability theory of differential equations and proved that the disease-free equilibrium is asymptotically stable if $R_{0}<1$, and the intrinsic growth rate of the Vibrio cholerae bacterium population is less than its natural death rate. The center manifold theory is applied to show the existence of forward bifurcation at the point $R_{0}=1$ and the local stability of endemic equilibrium if $R_{0}>1$. Furthermore, the performed numerical simulation results show that, as the rank of control measures applied increases from no control, weak control, and strong control measures, the recovered individuals are 55.02, 67.47, and 674.7, respectively. Numerical simulations are plotted using MATLAB software package.
\end{abstract}

\section{Introduction}

Cholera is a waterborne acute gastrointestinal infection characterized by diarrhea and vomiting, which kills within an hour if not treated [1]. Cholera is caused by drinking water or eating food contaminated by a bacterium called Vibrio cholerae ( $V$. cholerae) $[2,3]$. Most of the diseases are controlled by public health organizations including cholera which is characterized with severe vomiting and diarrhea [4]. The incubation period of cholera is less than five days [5]. However, cholera outbreak occurs twice in endemic areas, and it becomes the major issue in developing countries. Cholera infection can be transmitted from human to human or human to environment through effective contacts with bacterium $V$. cholerae [6].

Different mathematical models have been carried out to describe the transmission dynamics of cholera infection $[7,8]$. Codeco developed the first basic mathematical model for cholera infection. Furthermore, a new fractional derivative was developed by Hattaf [9] and applied to analyze the memory effect on the HIV-infected population. However, the new fractional derivative has not been applied to study the cases in the cholera infection.

In this study, we modified the model developed in [5], we focus on the logistic growth model of Vibrio cholerae concentration in reserved freshwater used for drinking purposes, and the contribution of infected individuals to the environment is assumed to be properly managed. That is, all waste disposals from cholera-infected patients are properly managed, and the contributions of humans to the environment are restricted. Hence, we developed an optimal control mathematical model that takes into consideration the loss of immunity after cholera infection recovery. Our model considers the reimmunization system for those individuals that lose immunity. Furthermore, we have applied a new fractional derivative and analyzed the relationship between the order of the derivative and the extinction status of cholera infection. 


\section{Mathematical Model Developments}

In this study, we have developed the deterministic mathematical model of cholera with optimal control strategies. We have divided the total population as (i) susceptible $(S)$ : these are individuals free of the infection but can be infected with the infection if exposed to the infectious people or environment contaminated with the cholera-causing bacterium; (ii) infected $(I)$ : these are individuals that get infected with cholera and can transmit infection to others with possible contacts that cause infection; (iii) recovered $(R)$ : these are individuals recovered from the cholera infection with treatment. The recovered individuals who lose immunity become susceptible; (iv) concentration of the Vibrio cholerae bacterium $B(t)$ : this covers an environment contaminated with Vibrio cholerae that cause the cholera epidemic.

Furthermore, the following assumptions are incorporated into the formulation of the model:

(i) The total population is considered to be nonconstant

(ii) All humans die due to cholera at the disease-induced death rate of $\varphi$

(iii) All waste products contain Vibrio cholerae which are assumed to be managed, and there is no contribution of the infected person to reserved freshwater

(iv) All human populations are assumed to die at the natural death rate $\mu$

(v) All new infected human individuals are recruited to the susceptible at the recruitment rate $\lambda$

(vi) Treatment is performed in reserved freshwater

(vii) Cholera-infected individuals recover at a recovery rate of $\eta$

(viii) Cholera infection-recovered individuals lose immunity at the rate $\gamma$

(ix) Cholera infection is transmitted from human to human at the rate $\beta$

(x) $V$. cholerae is ingested from the environment at the ingestion rate $\beta_{c}$

(xi) The saturated incidence rate is used in the transmission of cholera infection from the environment to humans

(xii) The concentration of $V$. cholerae in water that causes $50 \%$ chance of cholera infection is denoted by $\kappa$

(xiii) The carrying capacity of $V$. cholerae is $K$ (xiv) $V$. cholerae die at the rate of $v$

(xv) Cholera infection transmission preventive control effort is $u_{1}$

(xvi) Immunity loss control effort is $u_{2}$

(xvii) $V$. cholerae clearing control effort is $u_{3}$

In general, the stated assumptions can be described by the flow diagram.

The behavior of the population described in Figure 1 can be described by the mathematical model as

$$
\begin{aligned}
& \frac{\mathrm{d} S}{\mathrm{~d} t}=\lambda-\left(1-u_{1}\right)\left(\frac{\beta_{c} \mathrm{SB}}{k+B}+\beta \mathrm{SI}\right)+\left(1-u_{2}\right) \gamma R-\mu \mathrm{S}, \\
& \frac{\mathrm{d} I}{\mathrm{~d} t}=\left(1-u_{1}\right)\left(\frac{\beta_{c} \mathrm{SB}}{k+B}+\beta \mathrm{SI}\right)-(\eta+\varphi+\mu) I, \\
& \frac{\mathrm{d} R}{\mathrm{~d} t}=\eta I-\left(\left(1-u_{2}\right) \gamma+\mu\right) R, \\
& \frac{\mathrm{d} B}{\mathrm{~d} t}=\left(1-u_{3}\right) r B\left(1-\frac{B}{K}\right)-v B,
\end{aligned}
$$

with nonnegative initial conditions.

\section{Mathematical Analysis of Only Cholera Model without Optimal Control}

Formulated model (1) of cholera without control measures is reduced to the following form:

$$
\begin{aligned}
\frac{\mathrm{d} S}{\mathrm{~d} t} & =\lambda-\frac{\beta_{c} \mathrm{SB}}{k+B}-\beta \mathrm{SI}+\gamma R-\mu \mathrm{S}, \\
\frac{\mathrm{d} I}{\mathrm{~d} t} & =\frac{\beta_{c} \mathrm{SB}}{k+B}+\beta \mathrm{SI}-(\eta+\varphi+\mu) I, \\
\frac{\mathrm{d} R}{\mathrm{~d} t} & =\eta I-(\gamma+\mu) R, \\
\frac{\mathrm{d} B}{\mathrm{~d} t} & =r B\left(1-\frac{B}{K}\right)-\nu B,
\end{aligned}
$$

with nonnegative initial conditions.

\subsection{Invariant Regions}

Theorem 1. Solutions of model (1) are invariant in the region $\Omega \subset \mathfrak{R}_{+}^{4}$ such that

$$
\Omega=\Omega_{H} \times \Omega_{B} \subset \mathfrak{R}_{+}^{3} \times \mathfrak{R}_{+}=\left\{(S, I, R, B):(S, I, R) \in \mathfrak{R}_{+}^{3}, B \in \mathfrak{R}_{+}, N_{h}=S+I+R \leq \frac{\lambda}{\mu}, B \leq K\right\} .
$$

Proof. As in [10], to prove this theorem, we add the first three equations of model (1) so that

$$
\frac{\mathrm{d} N_{h}}{\mathrm{~d} t}=\lambda-\mu N_{h}-\varphi I \leq \lambda-\mu N_{h}
$$




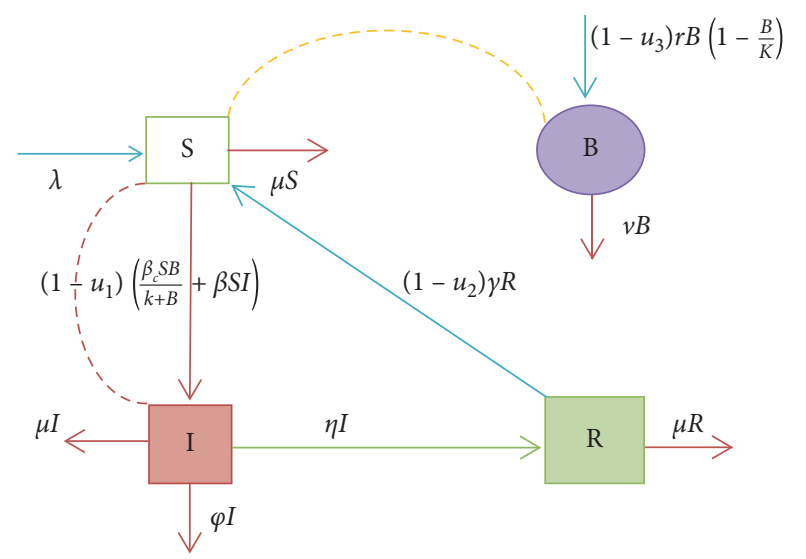

FIGURE 1: The flow diagram of the SIR-B model of the cholera epidemic with control measures.

Applying a definite integral over time interval $[0, t]$, the preceding inequality gives

$$
N_{h} \leq \frac{\lambda}{\mu}-\left(\frac{\lambda}{\mu}-N(0)\right) e^{-\mu t}
$$

Here, as time $t$ gets larger and larger, $N_{h}$ approaches to least upper bound $\lambda / \mu$. Also, $N_{h} \leq \lambda / \mu$, for all time $t$. Similarly, from the last equation of model (2), we obtain $B \leq K$ for all time $t$. Therefore, the invariant region $\Omega \subset \mathfrak{R}_{+}^{4}$ is given by

$$
\Omega=\left\{(S, I, R, B): N_{h}=S+I+R \leq \frac{\lambda}{\mu}, B \leq K\right\} .
$$

\subsection{Positivity Property of Solutions}

Theorem 2. For given positive initial conditions, all possible solutions of model (1) are positive in the invariant $\Omega \subset \Re_{+}^{4}$ such that

$$
\Omega=\{(S, I, R, B): S(0)>0, I(0) \geq 0, R(0) \geq 0, B(0) \geq 0\} .
$$

Proof. To prove the positivity property, we apply the methods employed in [11]. Consider the first equation of model (1):

$$
\frac{\mathrm{d} S}{\mathrm{~d} t}=\lambda-\frac{\beta_{c} \mathrm{SB}}{k+B}-\beta \mathrm{SI}+\gamma R-\mu \mathrm{S} .
$$

Rearranging the preceding equation, we get

$$
\frac{\mathrm{d} S}{\mathrm{~d} t}+\left(\frac{\beta_{c} B}{k+B}+\beta I+\mu\right) S=\lambda+\gamma R .
$$

Also, we have

$$
\begin{aligned}
\frac{\mathrm{d}}{\mathrm{d} t}\left[S(t) \exp \left\{\mu t+\int_{0}^{t}\left(\frac{\beta_{c} B(\zeta)}{k+B(\zeta)}+\beta I(\zeta)\right) \mathrm{d} \zeta\right\}\right] \\
=(\lambda+\gamma R) \exp \left\{\mu t+\int_{0}^{t}\left(\frac{\beta_{c} B(\zeta)}{k+B(\zeta)}+\beta I(\zeta)\right) \mathrm{d} \zeta\right\} .
\end{aligned}
$$

Applying integration on the preceding equation over time interval $[0, t]$, we get

$$
\begin{aligned}
& S(t) \exp \left\{\mu t+\int_{0}^{t}\left(\frac{\beta_{c} B(\zeta)}{k+B(\zeta)}+\beta I(\zeta)\right) \mathrm{d} \zeta\right\}-S(0) \\
& \quad=\int_{0}^{t}(\lambda+\gamma R(\zeta)) \exp \left\{\mu t+\int_{0}^{t}\left(\frac{\beta_{c} B(\zeta)}{k+B(\zeta)}+\beta I(\zeta)\right) \mathrm{d} \zeta\right\} \mathrm{d} t .
\end{aligned}
$$

Hence,

$$
\begin{aligned}
S(t)= & S(0) \exp \left[-\left\{\mu t+\int_{0}^{t}\left(\frac{\beta_{c} B(\zeta)}{k+B(\zeta)}+\beta I(\zeta)\right) \mathrm{d} \zeta\right\}\right]+\exp \left[-\left\{\mu t+\int_{0}^{t}\left(\frac{\beta_{c} B(\zeta)}{k+B(\zeta)}+\beta I(\zeta)\right) \mathrm{d} \zeta\right\}\right] \\
& \cdot\left[\int_{0}^{t}(\lambda+\gamma R(\zeta)) \exp \left\{\mu t+\int_{0}^{t}\left(\frac{\beta_{c} B(\zeta)}{k+B(\zeta)}+\beta I(\zeta)\right) \mathrm{d} \zeta\right\} \mathrm{d} t\right]>0 .
\end{aligned}
$$

Since the expressions

$$
\begin{aligned}
\lambda+\gamma R(\zeta)>0, & \exp \left[-\left\{\mu t+\int_{0}^{t}\left(\frac{\beta_{c} B(\zeta)}{k+B(\zeta)}+\beta I(\zeta)\right) \mathrm{d} \zeta\right\}\right]+\exp \left[-\left\{\mu t+\int_{0}^{t}\left(\frac{\beta_{c} B(\zeta)}{k+B(\zeta)}+\beta I(\zeta)\right) \mathrm{d} \zeta\right\}\right], \\
& \cdot\left[\int_{0}^{t}(\lambda+\gamma R(\zeta)) \exp \left\{\mu t+\int_{0}^{t}\left(\frac{\beta_{c} B(\zeta)}{k+B(\zeta)}+\beta I(\zeta)\right) \mathrm{d} \zeta\right\} \mathrm{d} t\right]
\end{aligned}
$$

are positive, the state variable $S(t)>0$ for all time $t$. Similarly, all other state variables are positive. Therefore, the set that contains all solution variables of model (1) is a positively invariant set. 
3.3. Disease-Free Equilibrium (DFE). The disease-free equilibrium $\left(E_{0}\right)$ of model ( 1 ) is given by

$$
E_{0}=\left(\frac{\lambda}{\mu}, 0,0,0\right) .
$$

3.4. Endemic Equilibrium (EE). The computed endemic equilibrium $E_{1}=\left(S^{*}, I^{*}, R^{*}, B^{*}\right)$ of model (2) is given by

$$
E_{1}=\left(S^{*}, I^{*}, \frac{\eta}{\gamma+\mu} I^{*}, B^{*}\right)
$$

where $I^{*}=\lambda-\mu S^{*} / \eta+\varphi+\mu, B^{*}=K(1-\nu / r)=B$, and $S^{*}=S$ is the solution of the equation

$$
\frac{\mu^{2} R_{0}}{\lambda} S^{2}-\left(\frac{\beta_{c} B}{K+B}+\mu\left(R_{0}+1\right)\right) S+\lambda=0 .
$$

3.5. Reproduction Number. In this section, the reproduction number $\left(R_{0}\right)$ is computed by employing the nextgeneration matrix $\left(\mathrm{FV}^{-1}\right)$ stated in research works $[1,5,12]$. Thus, from model $(2)$, the reproduction number can be computed as

$$
R_{0}=\rho\left(\mathrm{FV}^{-1}\right)
$$

$\underset{\text { whence, }}{\text { Hence }}=\left[\begin{array}{cc}\beta \lambda / \mu & \beta_{c} \lambda / \mu k \\ 0 & r\end{array}\right]$ and $V=\left[\begin{array}{cc}\eta+\varphi+\mu & 0 \\ 0 & \nu\end{array}\right]$.

$$
\mathrm{FV}^{-1}=\left[\begin{array}{cc}
\frac{\beta \lambda}{b \mu} & \frac{\beta_{c} k \lambda}{\mu \nu} \\
0 & \frac{r}{\nu}
\end{array}\right] .
$$
are

The eigenvalues of the preceding next-generation matrix

$$
\lambda_{1}=\beta \lambda / b \mu, \lambda_{2}=r / \nu
$$

Therefore,

$$
R_{0}=\max \left\{\frac{\beta \lambda}{b \mu}, \frac{r}{v}\right\}=\frac{\beta \lambda}{b \mu}
$$

where $b=\eta+\varphi+\mu$.

\subsection{Local Stability of Disease-Free Equilibrium}

Theorem 3. The disease-free equilibrium $\left(E_{0}\right)$ of model (2) is locally asymptotically stable for the reproduction number less than one and intrinsic growth rate $(r)$ of bacteria less than the natural death rate. On the contrary, $E_{0}$ is unstable for the reproduction number greater than one.

Proof. To approve the local stability of $E_{0}$, we compute the eigenvalues of the Jacobian matrix obtained from model (2) and evaluated at $E_{0}$. Hence, it follows that

$$
J\left(E_{0}\right)=\left[\begin{array}{cccc}
-\mu & -\frac{\beta \lambda}{\mu} & \gamma & -\frac{\beta_{c} \lambda}{\mu k} \\
0 & (\eta+\varphi+\mu)\left(R_{0}-1\right) & 0 & \frac{\beta_{c} \lambda}{\mu k} \\
0 & 0 & -(\gamma+\mu) & 0 \\
0 & 0 & 0 & r-\nu
\end{array}\right] .
$$

The computed eigenvalues of the preceding matrix are given by

$$
\lambda_{1}=-\mu, \lambda_{2}=-\mu, \lambda_{3}=(\eta+\varphi+\mu)\left(R_{0}-1\right), \lambda_{4}=r-\nu .
$$

From the stability principle on the eigenvalues computed from the Jacobian matrix evaluated at $E_{0}$, we conclude that the disease-free equilibrium $\left(E_{0}\right)$ is locally asymptotically stable if $R_{0}<1$ and $r<\nu$ and unstable if either $R_{0}>1$ or $r>v$.

3.7. Global Stability of Disease-Free Equilibrium. Model (2) can be rewritten in the following form:

$$
\begin{aligned}
& \frac{\mathrm{d} X}{\mathrm{~d} t}=H(X, Y), \\
& \frac{\mathrm{d} Y}{\mathrm{~d} t}=G(X, Y), G(X, 0)=0 .
\end{aligned}
$$
is

The disease-free equilibrium $E_{0}^{1}$ of the preceding system

$$
E_{0}^{1}=\left(X^{0}, 0\right)
$$

where $X^{0}$ is the disease-free equilibrium of the disease-free system.

According to Biswas et al. [13], to guarantee global asymptotic stability, we verify the following conditions $\mathrm{H} 1$ and $\mathrm{H} 2$ to be satisfied:

$\mathrm{H} 1$ : for $\mathrm{d} X / \mathrm{d} t=H(X, 0), X^{0}$ is the globally asymptotically stable equilibrium

H2: $G(X, Y)=P Y-\widehat{G}(X, Y), \widehat{G}(X, Y) \geq 0$ for $\quad(X$, $Y) \in \Omega$

Here, $P=D_{Y} G(X, 0)$ satisfy the condition of the Metzler matrix, and $\Omega$ is a region of feasible solutions. Now, we state the following theorem.

Theorem 4. The disease-free equilibrium of the cholera dynamic model is globally asymptotically stable if $R_{0}<1$ and $r<v$ if conditions $H 1$ and $H 2$ are satisfied and unstable whenever $R_{0}>1$.

Proof. From model (2), we have 


$$
H(X, 0)=\lambda-\mu S=H(S, 0) .
$$

Solving $H(X, 0)=0$, we obtain $S=\lambda / \mu$. Hence, $X^{0}=(\lambda / \mu, 0)$.

Here, $X^{0}$ is the globally stable equilibrium of equation

$$
\frac{\mathrm{d} X}{\mathrm{~d} t}=H(X, 0) \text {. }
$$

From the infected compartments of model (2), we obtain

$$
G(X, Y)=\left[\begin{array}{cc}
-(\eta+\varphi+\mu) & 0 \\
0 & r-v
\end{array}\right]\left[\begin{array}{l}
I \\
B
\end{array}\right]-\left[\begin{array}{c}
-\frac{\beta_{c} S B}{k+B}-\beta S I \\
\frac{r B^{2}}{K}
\end{array}\right] .
$$

At the disease-free equilibrium, the preceding equation reduces to the following form:

$$
\begin{aligned}
G(X, Y) & =G(X, Y)=\left[\begin{array}{cc}
-(\eta+\varphi+\mu) & 0 \\
0 & r-v
\end{array}\right]\left[\begin{array}{l}
I \\
B
\end{array}\right]-\left[\begin{array}{l}
0 \\
0
\end{array}\right] \\
& =\mathrm{PY}-\widehat{G}(X, Y),
\end{aligned}
$$

where $P=\left[\begin{array}{cc}-(\eta+\varphi+\mu) & 0 \\ 0 & r-\nu\end{array}\right]$ and $\widehat{G}(X, Y)=\left[\begin{array}{l}0 \\ 0\end{array}\right] \geq 0$,

Now, it follows that the formulated model satisfied the hypothesis conditions required as $G(X, Y)=P Y-\widehat{G}(X, Y)$, where $\widehat{G}(X, Y) \geq 0, \forall X, Y$. Therefore, $E_{0}$ is globally asymptotically stable if $R_{0}<1$.

\subsection{Bifurcation Analysis}

Theorem 5. The endemic equilibrium $\left(E_{1}\right)$ of model (2) exhibits backward bifurcation at $R_{0}=1$.

Proof. Let $\beta^{*}$ be a bifurcation parameter corresponding to parameter $\beta$ and obtained by setting $R_{0}=1$ and solving for $\beta^{*}$. Therefore, the bifurcation parameter $\left(\beta^{*}\right)$ is given by

$$
\beta^{*}=\frac{\mu(\eta+\varphi+\mu)}{\lambda} .
$$

To determine types of bifurcation, we construct Jacobian matrix $J\left(E_{0}, \beta^{*}\right)$ from model (2) and compute the eigenvalues of $J\left(E_{0}, \beta^{*}\right)$. Thus, we have

$$
J\left(E_{0}, \beta^{*}\right)=\left[\begin{array}{cccc}
-\mu-(\eta+\varphi+\mu) & \gamma & -\frac{\beta_{c} \lambda}{\mu k} \\
0 & 0 & 0 & \frac{\beta_{c} \lambda}{\mu k} \\
0 & 0 & -(\gamma+\mu) & 0 \\
0 & 0 & 0 & r-\nu
\end{array}\right] .
$$

The computed eigenvalues of the preceding matrix are given by

$$
\lambda_{1}=-\mu, \lambda_{2}=0, \lambda_{3}=-\mu, \lambda_{4}=r-\nu .
$$

Here, all eigenvalues are negative except $\lambda_{2}$ which is simply 0 with condition $r<v$. Therefore, according to Akanni et al. [14], model (2) exhibits bifurcation at bifurcation parameter $\beta^{*}$. To determine the type of bifurcation, we compute left eigenvector $v=\left(v_{1}, v_{2}, v_{3}, v_{4}\right)$ and right eigenvector $w=$ $\left(w_{1}, w_{2}, w_{3}, w_{4}\right)$ that satisfy the conditions $v \cdot J\left(E_{0}, \beta^{*}\right)=$ $0, J\left(E_{0}, \beta^{*}\right) \cdot w=0$, and $v \cdot w=1$. Hence, the computed left eigenvalues from equation $v \cdot J\left(E_{0}, \beta^{*}\right)=0$ are given by

$$
v_{1}=0, v_{2}=v_{2}, v_{3}=0, v_{4}=-\frac{\mu k}{\beta_{c} \lambda}(r-v) \text {. }
$$

And the computed right eigenvalues from equation $J\left(E_{0}, \beta^{*}\right) \cdot w=0$ are given by

$$
w_{1}=-\frac{(\eta+\varphi+\mu) w_{2}}{\mu}, w_{2}=w_{2}, w_{3}=0, w_{4}=0 .
$$

Also, from the condition $v \cdot w=1$, we have

$$
v_{2}=\frac{1}{w_{2}} .
$$

Let us choose $v_{2}=w_{2}=1$; we compute the following second-order partial derivatives of functions evaluated at the disease-free equilibrium and bifurcation parameter.

Now, set $x=\left(x_{1}, x_{2}, x_{3}, x_{4}\right)=(S, I, R, B)$ and

$$
f(x)=\left(\begin{array}{c}
f_{1} \\
f_{2} \\
f_{3} \\
f_{4}
\end{array}\right)(x)=\left(\begin{array}{c}
\lambda-\frac{\beta_{c} x_{1} x_{4}}{k+x_{4}}-\beta x_{1} x_{2}+\gamma x_{3}-\mu \mathrm{x}_{1} \\
\frac{\beta_{c} x_{1} x_{4}}{k+x_{4}}+\beta x_{1} x_{2}-(\eta+\varphi+\mu) x_{2} \\
\eta x_{2}-(\gamma+\mu) x_{3} \\
r x_{4}\left(1-\frac{x_{4}}{K}\right)-v x_{4}
\end{array}\right),
$$




$$
\begin{aligned}
\frac{\partial^{2} f_{1}}{\partial x_{1}^{2}} & =0, \frac{\partial^{2} f_{1}}{\partial x_{2}^{2}}=0, \frac{\partial^{2} f_{1}}{\partial x_{3}^{2}}=0, \frac{\partial^{2} f_{1}}{\partial x_{4}^{2}}=\frac{2 \beta_{c} k x_{1}}{\left(k+x_{4}\right)^{3}} \frac{\partial^{2} f_{2}}{\partial x_{1}^{2}}=0, \frac{\partial^{2} f_{2}}{\partial x_{2}^{2}}=0, \frac{\partial^{2} f_{2}}{\partial x_{3}^{2}}=0, \frac{\partial^{2} f_{2}}{\partial x_{4}^{2}}=-\frac{2 \beta_{c} k x_{1}}{\left(k+x_{4}\right)^{3}}, \\
\frac{\partial^{2} f_{3}}{\partial x_{1}^{2}} & =0, \frac{\partial^{2} f_{3}}{\partial x_{2}^{2}}=0, \frac{\partial^{2} f_{3}}{\partial x_{3}^{2}}=0, \frac{\partial^{2} f_{3}}{\partial x_{4}^{2}}=0, \frac{\partial^{2} f_{4}}{\partial x_{1}^{2}}=0, \frac{\partial^{2} f_{4}}{\partial x_{2}^{2}}=0, \frac{\partial^{2} f_{4}}{\partial x_{3}^{2}}=0, \frac{\partial^{2} f_{4}}{\partial x_{4}^{2}}=-\frac{2 r}{k}, \\
\frac{\partial^{2} f_{1}}{\partial x_{2} \partial x_{1}} & =-\beta, \frac{\partial^{2} f_{1}}{\partial x_{3} \partial x_{1}}=0, \frac{\partial^{2} f_{1}}{\partial x_{4} \partial x_{1}}=-\frac{\beta_{c} k}{\left(k+x_{4}\right)^{2}}, \frac{\partial^{2} f_{2}}{\partial x_{2} \partial x_{1}}=\beta, \frac{\partial^{2} f_{2}}{\partial x_{3} \partial x_{1}}=0, \frac{\partial^{2} f_{2}}{\partial x_{4} \partial x_{1}}=\frac{\beta_{c} k}{\left(k+x_{4}\right)^{2}}, \\
\frac{\partial^{2} f_{3}}{\partial x_{2} \partial x_{1}} & =0, \frac{\partial^{2} f_{3}}{\partial x_{3} \partial x_{1}}=0, \frac{\partial^{2} f_{3}}{\partial x_{4} \partial x_{1}}=0, \frac{\partial^{2} f_{4}}{\partial x_{2} \partial x_{1}}=0, \frac{\partial^{2} f_{4}}{\partial x_{3} \partial x_{1}}=0, \frac{\partial^{2} f_{4}}{\partial x_{4} \partial x_{1}}=0, \\
\frac{\partial^{2} f_{2}}{\partial x_{1} \partial \beta_{c}^{*}} & =\frac{\left(k x_{2}+x_{2} x_{4}+x_{4}\right)}{k+x_{4}}, \frac{\partial^{2} f_{2}}{\partial x_{2} \partial \beta^{*}}=x_{1}, \frac{\partial^{2} f_{2}}{\partial x_{3} \partial \beta_{c}^{*}}=0, \frac{\partial^{2} f_{2}}{\partial x_{4} \partial \beta_{c}^{*}}=\frac{k x_{1}}{\left(k+x_{4}\right)^{2}} .
\end{aligned}
$$

Next, the bifurcation coefficients $a$ and $b$ can be computed as follows:

$$
\begin{aligned}
& a=\sum_{k, i, j=1}^{4} v_{k} w_{i} w_{j} \frac{\partial^{2} f_{k}}{\partial x_{i} \partial x_{j}}\left(E_{0}, \beta^{*}\right)=-\frac{2 \beta_{c} \lambda}{\mu k^{2}}-\frac{2(\eta+\varphi+\mu) \beta_{c}}{\mu}<0, \\
& b=\sum_{k, i=1}^{4} v_{k} w_{i} \frac{\partial^{2} f_{k}}{\partial x_{i} \partial \beta^{*}}\left(E_{0}, \beta^{*}\right)=v_{2} w_{2} x_{1}=\frac{\lambda}{\mu}>0 .
\end{aligned}
$$

Hence, according to Akanni et al. [14], the model shows supercritical (forward) bifurcation at $R_{0}=1$.

\subsection{Stability of Endemic Equilibrium $\left(E_{1}\right)$}

Theorem 6. Endemic equilibrium is locally asymptotically stable if $R_{0}>1$.

Proof. It follows from the center manifold theory, a model that shows forward bifurcation has endemic equilibrium that exhibits locally asymptotic stability if $R_{0}>1$ [14].

Theorem 7. Endemic equilibrium is globally asymptotically stable if $R_{0}>1$.

Proof. It follows from the center manifold theory, a model that shows forward bifurcation has endemic equilibrium that exhibits globally asymptotic stability if $R_{0}>1$ [14].

\section{Application of a New Generalized Fractional Derivative with the Nonsingular Kernel}

In this section, using the works done in [9], we study the dynamics of the human population with a new generalized definition of the fractional derivative with a nonnegative singular kernel in the presence of cholera infection. The rate of change of human population, from model (2), can be described as

$$
\begin{aligned}
\frac{\mathrm{d} S}{\mathrm{~d} t} & =\lambda-\frac{\beta_{c} \mathrm{SB}}{k+B}-\beta \mathrm{SI}+\gamma R-\mu \mathrm{S}, \\
\frac{\mathrm{d} I}{\mathrm{~d} t} & =\frac{\beta_{c} \mathrm{SB}}{k+B}+\beta \mathrm{SI}-(\eta+\varphi+\mu) I, \\
\frac{\mathrm{d} R}{\mathrm{~d} t} & =\eta I-(\gamma+\mu) R .
\end{aligned}
$$

Let $N_{h}(t)$ be the size of human populations at time $t$ and defined as

$$
N_{h}(t)=S(t)+I(t)+R(t) .
$$

Differentiating both sides of the preceding equation with respect to time $t$ and using (37), we get

$$
\frac{\mathrm{d} N_{h}}{\mathrm{~d} t}=\lambda-\mu \mathrm{N}_{\mathrm{h}}-\varphi \mathrm{I} \leq \lambda-\mu \mathrm{N}_{\mathrm{h}}
$$

Now, considering the upper bound, the preceding inequality reduces to the form

$$
\frac{\mathrm{d} N_{h}}{\mathrm{~d} t}=\lambda-\mu \mathrm{N}_{\mathrm{h}}
$$

The solution of the preceding equation over time interval $[0, t]$ is given by

$$
N_{h}=\frac{\lambda}{\mu}-\left(\frac{\lambda}{\mu}-N_{h}(0)\right) e^{-\mu t}
$$

Definition 1. (new generalized fractional derivative with nonsingular kernel; see [9]).

Let $\alpha \in[0,1), \beta, \gamma>0$, and $f \in H^{1}(a, b)$. In the sense of Caputo, the $\alpha$-order new generalized fractional derivative of function $f(t)$ with respect to the weight function $w(t)$ is defined as

$$
{ }^{C} D_{a, t, w}^{\alpha, \beta, \gamma} f(t)=\frac{N(\alpha)}{1-\alpha} \frac{1}{w(t)} \int_{a}^{t} E_{\beta}\left[-\mu_{\alpha}(t-x)^{\gamma}\right] \frac{\mathrm{d}}{\mathrm{d} x}(w f)(x) \mathrm{d} x,
$$

where $w \in C^{1}(a, b), w, w^{\prime}>0$ on $[a, b], N(\alpha)$ is a normalized function obeying $N(0)=N(1)=1, \mu_{\alpha}=\alpha / 1-\alpha$, 
and $E_{\beta}(t)=\sum_{k=0}^{+\infty} t^{k} / \Gamma(\beta k+1)$ is the Mittag-Leffler function of parameter $\beta$.

For simplicity, we symbolize ${ }^{C} D_{a, t, w}^{\alpha, \beta, \gamma} f(t)$ by $D_{a, t, w}^{\alpha, \beta, \gamma} f(t)$. Assuming that when a cholera infection spreads within a community, the individuals get knowledge about the infection, we replace the classical derivative by $D_{a, t, w}^{\alpha, \beta, \gamma}$. Then, (41) becomes

$$
D_{a, t, w}^{\alpha, \beta, \gamma} N_{h}(t)=\lambda-\mu \mathrm{N}_{\mathrm{h}}(\mathrm{t})
$$

Applying the Laplace transform to (43), we get

$$
\mathscr{L}\left\{w(t) D_{a, t, w}^{\alpha, \beta, \gamma} N_{h}(t)\right\}=\lambda \mathscr{L}\{w(t)\}-\mu \mathscr{L}\left\{w(t) N_{h}(t)\right\} .
$$

Using Theorem 2 [9], we have

$$
\begin{aligned}
\mathscr{L}\left\{w(t) N_{h}(t)\right\}= & \frac{N(\alpha) w(0) T(0) s^{\beta-1}}{[N(\alpha)+\mu(1-\alpha)] s^{\beta}+\alpha \mu} \\
& +\frac{\lambda(1-\alpha) s^{\beta}+\lambda \alpha}{[N(\alpha)+\mu(1-\alpha)] s^{\beta}+\alpha \mu} \mathscr{L}\{w(t)\} .
\end{aligned}
$$

Let $a_{\alpha}=N(\alpha)+\mu(1-\alpha)$. Then,

$$
\begin{aligned}
\mathscr{L}\left\{w(t) N_{h}(t)\right\}= & \frac{N(\alpha) w(0) N_{h}(0)}{\alpha_{\alpha}} \frac{s^{\beta-1}}{s^{\beta}+\alpha \mu / a_{\alpha}} \\
& +\frac{\lambda(1-\alpha)}{a_{\alpha}} \frac{s^{\beta-1}}{s^{\beta}+\alpha \mu / a_{\alpha}} s \mathscr{L}\{w(t)\} \\
& +\frac{\lambda \alpha}{a_{\alpha}} \frac{1}{s^{\beta}+\alpha \mu / a_{\alpha}} \mathscr{L}\{w(t)\} .
\end{aligned}
$$

Therefore,

$$
\begin{aligned}
\mathscr{L}\left\{w(t) N_{h}(t)\right\}= & \frac{N(\alpha) w(0) N_{h}(0)}{\alpha_{\alpha}} \mathscr{L}\left\{E_{\beta}\left(-\frac{\alpha \mu}{a_{\alpha}} t^{\beta}\right)\right\}+\frac{\lambda(1-\alpha)}{a_{\alpha}} \mathscr{L}\left\{E_{\beta}\left(-\frac{\alpha \mu}{a_{\alpha}} t^{\beta}\right)\right\}\left(\mathscr{L}\left\{w^{\prime}(t)\right\}+w(0)\right) \\
& -\frac{\lambda}{\mu} \mathscr{L}\left\{\frac{\mathrm{d}}{\mathrm{d} t} E_{\beta}\left(-\frac{\alpha \mu}{a_{\alpha}} t^{\beta}\right)\right\} \mathscr{L}\{w(t)\} .
\end{aligned}
$$

Applying inverse Laplace, the preceding equation reduces to

$$
w(t) N_{h}(t)=\frac{N(\alpha) w(0) N_{h}(0)}{\alpha_{\alpha}} E_{\beta}\left(-\frac{\alpha \mu}{a_{\alpha}} t^{\beta}\right)+\frac{\lambda(1-\alpha)}{a_{\alpha}} E_{\beta}\left(-\frac{\alpha \mu}{a_{\alpha}} t^{\beta}\right) w^{\prime(t)}-\frac{\lambda}{\mu} \frac{\mathrm{d}}{\mathrm{d} t} E_{\beta}\left(-\frac{\alpha \mu}{a_{\alpha}} t^{\beta}\right) w(t)+\frac{\lambda(1-\alpha) w(0)}{a_{\alpha}} E_{\beta}\left(-\frac{\alpha \mu}{a_{\alpha}} t^{\beta}\right)
$$

Letting $w(t)=1$ and applying the integration by parts, the preceding equation reduces to

$$
N_{h}(t)=\frac{\lambda}{\mu}-\frac{N(\alpha)}{a_{\alpha}}\left(\frac{\lambda}{\mu}-N_{h}(0)\right) E_{\beta}\left(-\frac{\alpha \mu}{a_{\alpha}} t^{\beta}\right)
$$

In the next section, using numerical simulations, we will analyze the impact of the order of the new fractional derivative on the behavior of solutions of the cholera infection model.

\section{Numerical Simulations}

The parameters presented in Table 1 are used in the simulation of solutions of the SIR-B model and are either assumed logically or taken from the literature.

\section{Results and Discussion}

In this study, the three-dimensional dynamical system is formulated and analyzed, considering the interaction of human to human and human to environment in the transmission of the Vibrio cholerae bacterium. The analysis of the SIR-B model of the cholera epidemic shows that we can control cholera more effectively if we apply strong effort on the preventive, sanitation, hygiene, vaccination, and follow-up program. Figure 2 shows that, without control measures, the recovered individuals and others become susceptible. Figure 3 shows that weak control measures do not bring a significant change on the epidemic even though the number of pathogens is reduced. Figure 4 shows that the high contribution of control measures helps in the reduction 
TABLE 1: Description of the parameter value and source.

\begin{tabular}{lcc}
\hline Parameter & Parameter value & Source \\
\hline$\lambda$ & $15 /$ day & {$[15]$} \\
$\beta_{c}$ & $1.7\left(10^{-8}\right)$ & {$[5]$} \\
$\beta$ & $1.48\left(10^{-8}\right)$ & {$[5]$} \\
$\gamma$ & $0.025 /$ day & {$[15]$} \\
$\eta$ & $0.004 /$ day & {$[15]$} \\
$\mu$ & $0.0000548 /$ day & {$[15]$} \\
$\varphi$ & $0.15 /$ day & {$[15]$} \\
$\nu$ & $0.033 /$ day & {$[15]$} \\
$\kappa$ & $10^{6}$ cells/ml & {$[15]$} \\
$r$ & 0.0004 & Estimated \\
$K$ & $10^{8}$ cells/ml & Estimated \\
\hline
\end{tabular}

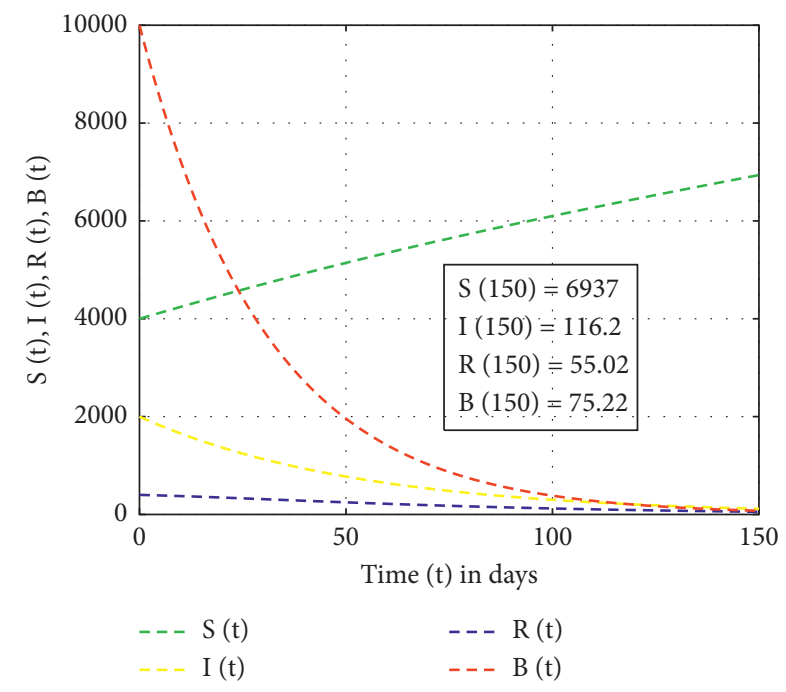

FIgURE 2: Simulation of the SIR-B model with $u_{1}=0$, $u_{2}=0$, and $u_{3}=0$.

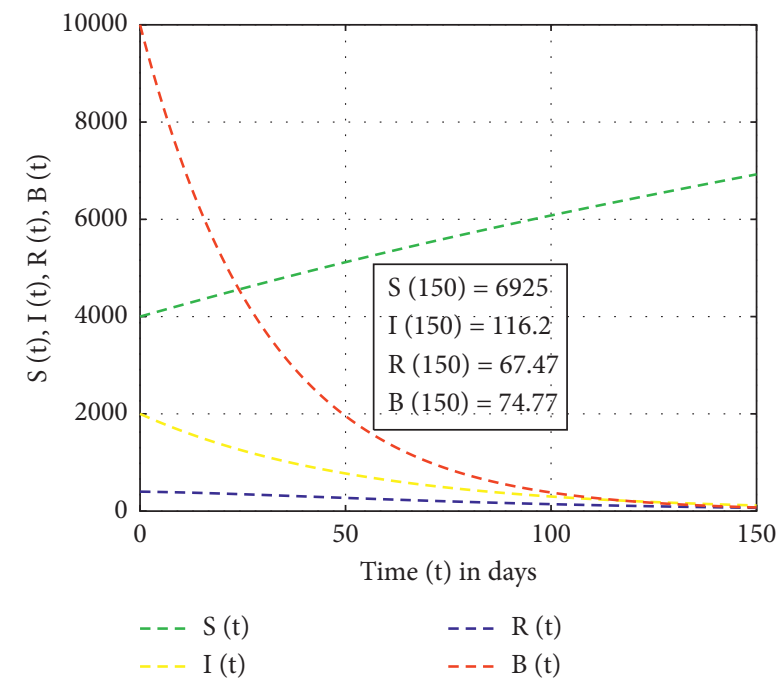

FIGURE 3: Simulation of the SIR-B model with weak control measures $\left(u_{1}=u_{2}=u_{3}=0.1\right)$.

of the Vibrio cholerae bacterium in freshwater but increases recovered individuals. Also, from Figures 2-4, one can observe that, as control measures increase applied as no

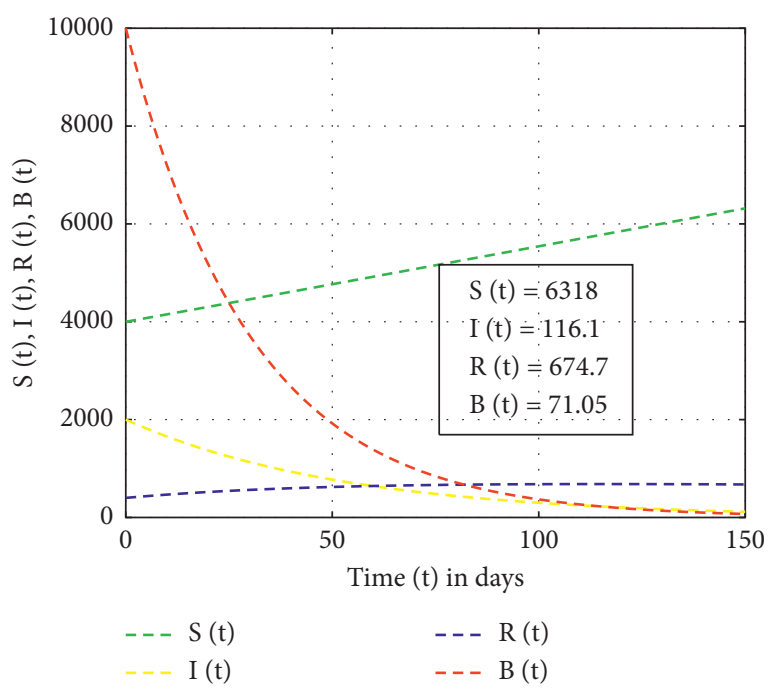

FIgURE 4: Simulation of the SIR-B model with strong control measures $\left(u_{1}=0.95, u_{2}=0.95\right.$, and $\left.u_{3}=0.95\right)$.

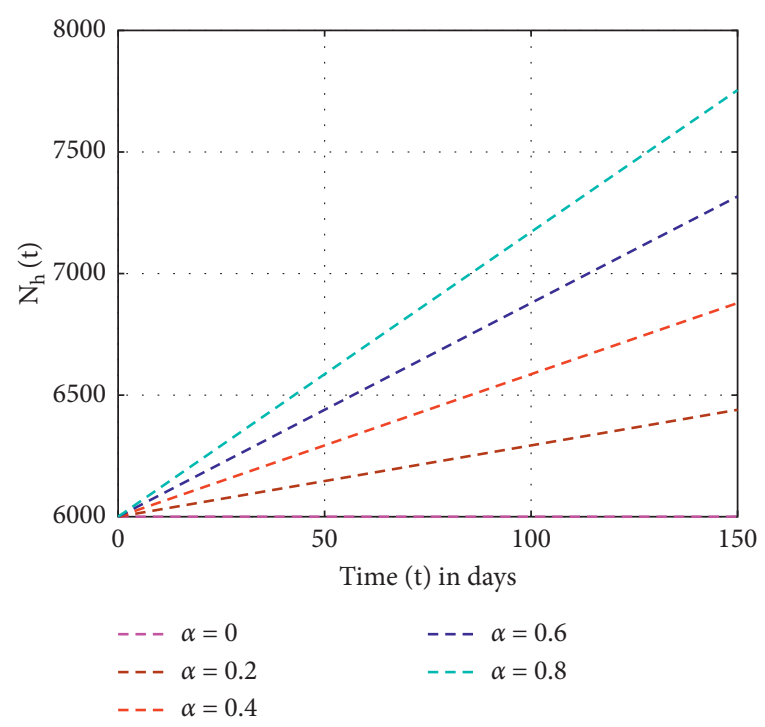

FIGURE 5: Simulation of total human population size described in (49) for different values of the fractional order ( $\alpha=0, \alpha=0.2, \alpha=0.4, \alpha=0.6$, and $\alpha=0.8)$ and $N_{h}(0)=6000$.

control, weak control, and strong control, the recovered individuals increase as 55.02, 67.47, and 674.7, respectively. The SIR-B model of cholera exhibits globally asymptotic stability at disease-free equilibrium if $R_{0}>1$ and unstable if $r>v$. Figure 5 describes that, as the order of fractional derivatives increases, the total population size approaches to disease-free equilibrium of the cholera model. This shows that, as the human population gets free of cholera, the behaviors of the population change along with the order of the new fractional derivative. Furthermore, the memory effect results we obtained in this study are supported with the study done in [9].

\section{Conclusion}

The current study pointed out that, as we apply strong control measures at all levels, the concentration of Vibrio 
cholerae in freshwater will be reduced. Furthermore, the study pointed out that, to significantly reduce the concentration of Vibrio cholerae in reserved freshwater, the intrinsic growth rate of the Vibrio cholerae bacterium must be less than the natural death rate of the Vibrio cholerae bacterium in freshwater. The stability of the disease-free equilibrium SIR-B model is accompanied with the intrinsic growth rate of Vibrio cholerae that needs to be less than its natural death rate and reproduction number less than one. The new fractional derivative results in that the memory effect in the human population has a direct relationship. As the order of the new fractional derivative increases, human memories of cholera infection controlling also increase.

\section{Data Availability}

No data were used to support this study.

\section{Disclosure}

This article is a part of a $\mathrm{PhD}$ thesis at Wollega University.

\section{Conflicts of Interest}

The authors declare that there are no conflicts of interest.

\section{Acknowledgments}

The first author would like to thank Hawassa College of Teacher Education, Ministry of Science and Higher Education, and Wollega University for their support to join the $\mathrm{PhD}$ program and continue in the research work.

\section{References}

[1] J. Lin, R. Xu, and X. Tian, “Transmission dynamics of cholera with hyperinfectious and hypoinfectious vibrios: mathematical modelling and control strategies," Mathematical Biosciences and Engineering, vol. 16, no. 5, pp. 4339-4358, 2019.

[2] A. P. Lemos-Paião, C. J. Silva, and D. F. M. Torres, "A cholera mathematical model with vaccination and the biggest outbreak of world's history," AIMS Mathematics, vol. 3, no. 4, pp. 448-463, 2018.

[3] S. Liao, "Stability analysis and application of a mathematical cholera model," Mathematical Biosciences and Engineering, vol. 8, no. 3, pp. 733-752, 2011.

[4] F. Nyabadza, J. M. Aduamah, and J. Mushanyu, "Modelling cholera transmission dynamics in the presence of limited resources," BMC Research Notes, vol. 12, pp. 1-8, 2019.

[5] C. Yang and J. Wang, "On the intrinsic dynamics of bacteria in waterborne infections," Mathematical Biosciences, vol. 296, pp. 71-81, 2018.

[6] S. D. Hove-musekwa, F. Nyabadza, C. Chiyaka, P. Das, A. Tripathi, and Z. Mukandavire, "Modelling and analysis of the effects of malnutrition in the spread of cholera," Mathematical and Computer Modelling, vol. 53, no. 9-10, pp. 1583-1595, 2011.

[7] S. Mushayabasa and C. P. Bhunu, "BioSystems Is HIV infection associated with an increased risk for cholera ? Insights from a mathematical model," Biosystems, vol. 109, no. 2, pp. 203-213, 2012.
[8] P. van den Driessche, "Reproduction numbers of infectious disease models," Infectious Disease Modelling, vol. 2, no. 3, pp. 288-303, 2017.

[9] K. Hattaf, "A new generalized defifinition of fractional derivative with non-singular kernel," Computation, vol. 8, no. 2, p. $49,2020$.

[10] K. Hattaf, A. Lashari, Y. Louartassi, and N. Yousfi, "A delayed SIR epidemic model with general incidence rate," Electronic Journal of Qualitative Theory of Differential Equations, vol. 3, no. 3, pp. 1-9, 2013.

[11] S. Sharma and G. P. Samanta, "Dynamical behaviour of an HIV/AIDS epidemic model," Differential Equations and Dynamical Systems, vol. 22, no. 4, pp. 369-395, 2013.

[12] S. Sharma and G. P. Samanta, "Analysis of a chlamydia epidemic model," Journal of Biological Systems, vol. 22, no. 04, pp. 713-744, 2014.

[13] S. K. Biswas, U. Ghosh, and S. Sarkar, "Mathematical model of zika virus dynamics with vector control and sensitivity analysis," Infectious Disease Modelling, vol. 5, pp. 23-41, 2020.

[14] J. O. Akanni, S. Olaniyi, and F. O. Akinpelu, "Global asymptotic dynamics of a nonlinear illicit drug use system," Journal of Applied Mathematics and Computing, vol. 66, no. 12, pp. 39-60, 2020.

[15] E. A. Bakare and S. Hoskova-Mayerova, "Optimal control analysis of cholera dynamics in the presence of asymptotic transmission," Axioms, vol. 10, no. 2, p. 60, 2021. 